\title{
APPROXIMATION BY POLYNOMIALS IN TWO DIFFEOMORPHISMS
}

\author{
BY A. G. O'FARRELL AND K. J. PRESKENIS
}

We denote by $\mathbf{C}$ the complex plane. If $f$ and $g$ are complex-valued functions on a set $S$, then $\mathbf{C}[f, g]$ denotes the algebra of polynomials in $f$ and $g$, with complex coefficients, regarded as functions on $S$.

Theorem. Let $1 \leq k \in Z$, and let $f$ and $g$ be $C^{k}$ diffeomorphisms of $\mathbf{C}$ into $\mathbf{C}$, having opposite degrees. Then $\mathbf{C}[f, g]$ is dense in the Fréchet space $C^{k}(\mathbf{C})$, i.e., given $h \in C^{k}(\mathbf{C})$, and $X \subset \mathbf{C}$ compact, there is a sequence $h_{n} \in \mathbf{C}[f, g]$ such that $h_{n}$ and its derivatives up to order $k$ tend to $h$ and its derivatives, uniformly on $X$.

In case $f(z)=z$ and $g(z)=\bar{z}$, the Theorem reduces to a result of Weierstrass.

Since each diffeomorphism of the closed unit disc $D$ into $\mathbf{C}$ extends to a diffeomorphism of $\mathbf{C}$ into $\mathbf{C}$, we deduce the following.

Corollary. Let $f$ and $g$ be $C^{\mathbf{1}}$ diffeomorphisms of $D$ into $\mathbf{C}$, having opposite degrees. Then $\mathbf{C}[f, g]$ is dense in $C(D)$.

This settles an old chestnut in the field of uniform algebras. It remains open whether the Corollary works for $k=0$, i.e., for all pairs of homeomorphisms of opposite degrees.

Proof of TheOREM. Without loss of generality, we may take $g=z$, because the chain rule for $D^{j}(h \circ g)$ is linear in $h$ and involves only $D^{i} h$ and $D^{i} g$ for $0 \leq i \leq j$.

Since $f$ has degree -1 , we deduce that $\left|f_{\bar{z}}\right|>\left|f_{z}\right|$ on C. In particular, $f_{\bar{z}} \neq 0$, so the graph $G=\left\{(z, f(z)) \in \mathbf{C}^{2}: z \in \mathbf{C}\right\}$, which is a $C^{k}$ submanifold of $\mathbf{C}^{2}$, has no complex tangents. By the Range-Siu theorem [2], $C^{k}(G)$ is the closure of the space $O(G)$ of all functions holomorphic in a neighbourhood of $G$. If we can show that $G$ has an exhaustion by polynomially-convex compact sets, then by the functional calculus [4, Chapter 8], it will follow that $\mathbf{C}[z, w]$ is dense in $O(G)$, and hence in $C^{k}(G)$; since $z \mapsto(z, f)$ is a $C^{k}$ diffeomorphism of $\mathbf{C} \rightarrow G$, this will imply that $\mathbf{C}[z, f]$ is dense in $C^{k}(\mathbf{C})$. Thus it suffices to show that $X=\{(z, f(z)): z \in K\}$ is polynomially-convex whenever $K \subset \mathbf{C}$ is a closed disc.

Fix a closed disc $K \subset \mathbf{C}$. By modifying $f$ off $K$, if need be, we may assume $f$ maps $\mathbf{C}$ onto $\mathbf{C}$, that $D f$ and $D f^{-1}$ are bounded and uniformly continuous, and that $\left|f_{\bar{z}}\right|$ and $1-\left|f_{z} / f_{\bar{z}}\right|$ are bounded away from zero. We need two lemmas, which are essentially classical results of Wermer.

Received by the editors August 10, 1983.

1980 Mathematics Subject Classification. Primary 46J10; Secondary 30E10, 32E30. 
LEMMA 1. There exists a constant $\lambda_{1}>0$ such that

$$
(z-a)(f(z)-f(a))+\lambda f_{\bar{z}}(a)
$$

is nonzero whenever $0<\lambda<\lambda_{1}, a \in \mathbf{C}$, and $z \in \mathbf{C}$.

Proof. Pick $\delta>0$ such that the modulus of continuity $\omega(\delta)$ of $D f$ at $\delta$ is less than half $\left(\inf \left|f_{\bar{z}}\right|\right)\left(1-\sup _{\mathbf{C}}\left|f_{z} / f_{\bar{z}}\right|\right.$. Applying the mean value theorem to the real and imaginary parts of $f$ we deduce that for $0<|z-a|<\delta$, the value $f(z)-f(a)$ differs from $f_{\bar{z}}(a) \overline{(z-a)}+f_{z}(a)(z-a)$ by less than $2 \omega(\delta)|z-a|$. Thus

$$
\operatorname{Re} \frac{(z-a)(f(z)-f(a))}{f_{\bar{z}}(a)} \geq 0
$$

whenever $|z-a|<\delta$. But for $|z-a| \geq \delta$,

$$
\left|\frac{(z-a)(f(z)-f(a))}{f_{\bar{z}}(a)}\right| \geq \frac{\delta^{2}\left(\sup \left|D f^{-1}\right|\right)^{-1}}{\inf \left|f_{\bar{z}}\right|} .
$$

Denoting the right-hand side by $\lambda_{1}$, we see that $(z-a)(f(z)-f(a)) / f_{\bar{z}}(a)$ omits $\left\{-\lambda: 0<\lambda<\lambda_{1}\right\}$, for all $a$ and $z$, so the lemma is proved.

Let us denote the uniform closure of $\mathbf{C}[z, f]$ in $C(K)$ by $A$.

LEMMA 2. Suppose that for each $a \in K$, there exists a sequence $\lambda_{n} \downarrow 0$ such that $(z-a)(f(z)-f(a))+\lambda_{n} f_{\bar{z}}(a)$ is invertible in $A$. Then $A=C(K)$.

Proof. Briefly, let $\mu$ be a measure on $K$, annihilating $A$. It suffices to show that the Cauchy transform $\hat{\mu}(a)=\int d \mu(\zeta) / \zeta-a$ vanishes at every point $a \in K$ at which the Newtonian potential $\int d|\mu|(\varsigma) /|\zeta-a|$ is finite. But the hypothesis, together with Lemma 1 , yields a sequence $f_{n} \in A$ such that $f_{n} \rightarrow$ $(z-a)^{-1}$, pointwise on $K \sim\{a\}$, and $\left|f_{n}(z)\right| \leq$ const $|z-a|^{-1}$. Thus the dominated convergence theorem yields the desired result.

We remark that the hypothesis of Lemma 2 can be weakened to "almost all $a \in K^{\prime \prime}$.

Conclusion of Proof of Theorem. Suppose $X$ is not polynomiallyconvex. Then $A \neq C(K)$, so by Lemma 2, there exists $a \in K$ and $\lambda_{2}>0$ such that for every $\lambda$ with $0<\lambda<\lambda_{2}$, the polynomial $(z-a)(w-f(a))+\lambda f_{\bar{z}}(a)$ has a zero somewhere on the polynomially-convex hull of $X$. Fix $\lambda$, with $0<\lambda<\min \left\{\lambda_{1}, \lambda_{2}\right\}$. Then the family of algebraic curves

$$
(z-a-t)(w-f(a+t))+\lambda f_{\bar{z}}(a+t)=0 \quad(0 \leq t<\infty)
$$

is a curve of algebraic hypersurfaces which meets the hull of $X$, does not meet $X$ (by Lemma 1), and goes to the hyperplane at infinity (since $f$ maps onto $\mathbf{C}$, and $f_{\bar{z}}$ is bounded). This contradicts Oka's characterization of polynomial hulls, as given in [3, (1.2), p. 263]. Thus $X$ is polynomially-convex, and we are done.

We remark that minor modifications to the foregoing proof permit us to strengthen the Corollary, as follows:

Let $f$ be an orientation-reversing homeomorphism of $\mathbf{C}$ into $\mathbf{C}$, which is locally $C^{1}$ and noncritical off a closed set $E$, having area zero and not separating the plane. Then $\mathbf{C}[z, f]$ is dense in $C(\mathbf{C})$. 
Also, for any compact set $X$ in $\mathbf{C}$ and for $0<\alpha<1$, suppose $\operatorname{Lip}(\alpha, X)$ denotes the space of bounded functions $g$ of $X$ into $\mathbf{C}$ such that for some $K>0,|g(z)-g(w)| \leq K|z-w|^{\alpha}$ for all $z, w \in X$ with norm $\sup |g|+$ Least $K$ and suppose $\operatorname{lip}(\alpha, X)$ denotes those functions $g \in \operatorname{Lip}(\alpha, X)$ such that, given $\epsilon>0$, there exists $\delta>0$ such that $|g(z)-g(w)| \leq \epsilon|z-w|^{\alpha}$ whenever $z$ and $w$ satisfy $|z-w|<\delta$. In view of the results given in [1, p. 227], the conclusion of the above remark implies $\mathbf{C}[z, f]$ is dense in $\operatorname{lip}(\alpha, X)$ for any compact set $X$ in $\mathbf{C}$.

Finally, we remark that the Theorem of this paper is sharp in the sense that one critical point destroys it.

\section{REFERENCES}

!. A. G. O'Farrell and K. J. Preskenis, Approximation by polynomials in two complex variables, Math. Ann. 246 (1980), 225-232.

2. M. Range and Y.-T. Siu, $C^{k}$ approximation by holomorphic functions and $\bar{\partial}$-closed forms on $C^{k}$ submanifolds of a complex manifold, Math. Ann. 210 (1974), 105-122.

3. G. Stolzenberg, Polynomially and rationally convex sets, Acta Math. 109 (1963), 259289.

4. J. Wermer, Banach algebras and several complex variables, Springer, 1976.

Department of Mathematics, Maynooth College, Co. Kildare, Ireland

Department of Mathematics, Framingham State College, Framingham, MASSACHUSETTS 01701 
